LBNL-42045

CBP Note-259

\title{
Generation of Subpicosecond X-ray Pulses Using RF Orbit Deflection*
}

\author{
A. Zholents, P. Heimann, M. Zolotorev, and J. Byrd \\ Ernest Orlando Lawrence Berkeley National Laboratory \\ Berkeley, California 94720
}

Submitted to NIMA

July 1998

\footnotetext{
${ }^{*}$ This work was supported by the Director, Office of Energy Research, Office of High Energy and Nuclear Physics, Division of High Energy Physics, of the U.S. Department of Energy under Contract No. DE-AC 03-76SF00098.
} 


\title{
Generation of Subpicosecond X-ray Pulses Using RF Orbit Deflection
}

\author{
A. Zholents, P. Heimann, M. Zolotorev, and J. Byrd
}

\begin{abstract}
A technique is proposed for producing high average intensity $\mathrm{x}$-ray radiation from a storage ring for studies of the ultrafast phenomena on a subpicosecond time scale. Two RF cavity accelerating structures excited in the $E_{110}$ mode can be installed in a storage ring to create vertical displacements of electrons correlated with their longitudinal position in the bunch. The magnitude of these displacements can be sufficient for the $\mathrm{x}$-ray radiation of the electron bunch between accelerating structures to be viewed as produced by a large number of independent sources, each of a subpicosecond duration.
\end{abstract}

\section{Introduction}

Electron bunches circulating in a storage ring produce synchrotron light in pulses with a typical duration of a few tens of picoseconds defined by the electron bunch length. Although this is adequate for many applications, it is too long for a direct probing of the atomic motion associated with phase transitions, coherent vibrational motion and ultrafast chemical reactions, which require temporal resolution on the order of one vibration period of atoms (approximately 100 femtoseconds). Here we consider a technique where pulses of synchrotron radiation much shorter than a picosecond can be obtained without a reduction of the bunch length. We propose to do this by creating a condition when the radiation of electrons sitting in each subpicosecond slice of the bunch could be spatially separated from the radiation of electrons of the neighboring slices. Then we can either use the radiation of each slice individually or combine the radiation of all of them in one subpicosecond pulse. 


\section{The method}

The separation of subpicosecond electron bunch slices is made by creating a correlation between the longitudinal coordinates of the electrons within the electron bunch and their vertical angles. Consider an excitation of the first dipole mode $E_{110}$ of an RF cavity accelerating structure. The azimuthal and longitudinal components of this mode written near the coordinate center in the cylindrical coordinates $r, \phi, s$ have the form:

$$
\begin{aligned}
\mathcal{E}_{s} & =\mathcal{E}_{s 0} J_{1}(k r) \sin \phi \cos (\omega t+\psi), \\
H_{\phi} & =\mathcal{E}_{s 0} J_{1}^{\prime}(k r) \sin \phi \sin (\omega t+\psi),
\end{aligned}
$$

where $J_{1}$ is the Bessel function and $J_{1}^{\prime}$ is its derivative with respect to the argument, $k=2 \pi / \lambda_{r f}$ is the wave number, $\lambda_{r f}$ is the RF wave length, $\mathcal{E}_{s 0}$ is the amplitude value of the electric field and wand $\psi$ are the RF frequency and phase.

While passing the accelerating structure, electrons are deflected by the RF magnetic field an amount equal to:

$$
y^{\prime}(z)=\theta_{0} \sin (k z), \quad \theta_{0}=\frac{e U}{E_{b}},
$$

where $z$ is the electron longitudinal coordinate relative to the bunch center, $E_{b}$ is the beam energy, $e$ is the electron charge and $e U$ is the energy gain in the accelerating structure calculated at the coordinates with the maximum electric field. It is assumed that the RF phase is chosen in a such way that $\psi=0$ when the bunch center is at the center of the cavity.

This defiection couples the longitudinal and transverse motion of the electrons. In order to confine this coupling in a section of a storage ring, a second accelerating structure is needed. When placed an integer number of half betatron wavelengths downstream of the first accelerating structure, the second accelerating structure provides perfect compensation for all distortions to the longitudinal and transverse motion of the electrons made in the first accelerating structure. ${ }^{*}$

*A similar arrangement of two accelerating structures with $E_{110}$ operation mode was considered for monochromatization of the center-of-mass energy of colliding particles of counter-rotating beams in colliders [1] and for collisions with a crossing angle [2]. 


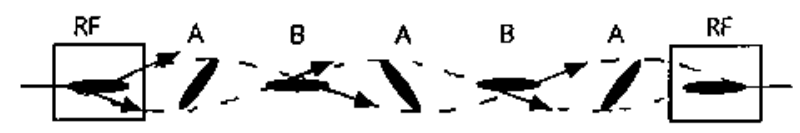

Figure 1: A schematic of the beam coupling produced by the RF cavities operated at $E_{110}$ mode.

Figure 1 schematically shows two accelerating structures, trajectories of the head and tail parts of the electron bunch and side views of the bunch profile in the several locations as it propogates from the left to the right. There are two types of locations along the orbit, which are convenient source points of synchrotron radiation for our purpose. At the A locations, the coordinate displacements of the electrons have reached their maximums. Instead, at the $\mathrm{B}$ locations, the variation in angle of the electron trajectories has its maximum value.

The correlated coordinate displacements of electrons in the A locations is written:

$$
\delta y(z)=\theta_{0} \sqrt{\beta_{r f} \beta} \sin (k z),
$$

where $\beta_{r f}$ is the vertical beta function at the RF cavity and $\beta$ is the vertical beta function in the A locations. The correlated angular displacements of electrons in the $\mathrm{B}$ locations is written:

$$
\delta y \prime(z)=\theta_{0} \sqrt{\frac{\beta_{r f}}{\beta}} \sin (k z)
$$

where $\beta$ is taken now in the $B$ locations.

For zero beam emittance we can say that the radiation of each subpicosecond slice is separated from the radiation of the neighboring slices if the difference in angle or coordinate between the neighboring slices is larger than 
the opening angle of the radiation, $\sigma_{r \prime}$, in the case of the angular separation or the diffraction-limited size of the radiation, $\sigma_{r}=\frac{\lambda}{4 \pi \sigma_{r}}$, in the case of coordinate separation. Both sizes $\sigma_{r}$ and $\sigma_{r}$ are taken at the source location. For a non-zero beam emittance we need to account for the broadening of the radiation field due to the coordinate and angular spread of electrons and consider $\left(\sigma_{y}^{2}+\sigma_{r}^{2}\right)^{1 / 2}$ and $\left(\sigma_{y^{\prime}}^{2}+\sigma_{r^{\prime}}^{2}\right)^{1 / 2}$ instead of $\sigma_{r}$ and $\sigma_{r^{\prime}}$, where $\sigma_{y}$ and $\sigma_{y \prime}$ are the vertical electron beam size and divergence at the source position. This broadening is much less in the vertical direction because vertical beam emittance in the storage ring is much smaller than the horizontal emittance.

Defining the length of the subpicosecond slice as $\sigma_{s}$ and the bunch length as $\sigma_{z}$, we can write a magnitude $\delta y\left(\sigma_{z}\right)$ in the A locations and a magnitude $\delta y^{\prime}\left(\sigma_{z}\right)$ in the B locations needed for a spatial separation of the radiation of $M=\sigma_{z} / \sigma_{s}$ subpicosecond slices:

$$
\begin{aligned}
\delta y\left(\sigma_{z}\right) & \geq M\left(\sigma_{y}^{2}+\sigma_{r}^{2}\right)^{1 / 2} \\
\delta y^{\prime}\left(\sigma_{z}\right) & \geq M\left(\sigma_{y^{\prime}}^{2}+\sigma_{r^{\prime}}^{2}\right)^{1 / 2} .
\end{aligned}
$$

In addition in the first case, one also needs to check that the vertical beam tilt is larger than the opening angle of the radiation, i.e. that $\arctan \left[\delta y\left(\sigma_{z}\right) / \sigma_{s}\right]>$ $\left(\sigma_{y \prime}^{2}+\sigma_{r \prime}^{2}\right)^{1 / 2}$. Usually this condition is easily satisfied along with the first Eq. (5).

Using Eqs. $(3,4)$ in the left parts of Eqs. (5), and assuming $k \sigma_{z} \leq 1$, we find the energy gain $e U$ required for the creation of $M$ subpicosecond slices:

$$
\begin{aligned}
& \frac{e U}{E_{b}} \geq \frac{M}{k \sigma_{z}} \sigma_{y^{\prime}}^{r f} \sqrt{1+\left(\frac{\sigma_{r}}{\sigma_{y}}\right)^{2}} \\
& \frac{e U}{E_{b}} \geq \frac{M}{k \sigma_{z}} \sigma_{y^{\prime}}^{r f} \sqrt{1+\left(\frac{\sigma_{r t}}{\sigma_{y^{\prime}}}\right)^{2}},
\end{aligned}
$$

where $\sigma_{y^{\prime}}^{r f}=\sqrt{\epsilon_{y} / \beta_{r f}}$ is the vertical angular beam size in the location of the RF cavity, and $\epsilon_{y}$ is the vertical beam emittance.

As a consequence of the correlated tilt to the electron bunches, one has to examine whether it affects the lifetime of the beam, i.e. whether there is enough of an aperture in the constrained locations (typically, the location of the undulators). Considering that the longitudinal extent of the beam is restricted by $\pm 7 \sigma_{z}$, which is usually sufficient to ensure a good lifetime, we can write:

$$
A_{y} \geq 20 \sigma_{y}+C O D+2 \delta y\left(7 \sigma_{z}\right)
$$


Table I: Examples of the coordinate and angular slicing

\begin{tabular}{|c|c|c|}
\hline Beam energy, $\mathrm{GeV}$ & 1.5 & 6.0 \\
\hline$\sigma_{z}, \mathrm{~cm}$ & 0.4 & 0.4 \\
\hline Number of slices, $M$ & 90 & 90 \\
\hline$\beta_{r f}, \mathrm{~m}$ & 100 & 100 \\
\hline $\mathrm{RF}$ wavelength, cm & 30 & 30 \\
\hline Vertical emittance, $\mathrm{m} \cdot \mathrm{rad}$ & $4 \times 10^{-11}$ & $4 \times 10^{-11}$ \\
\hline Horizontal emittance, $\mathrm{m} \cdot \mathrm{rad}$ & $4 \times 10^{-9}$ & $4 \times 10^{-9}$ \\
\hline Undulator period, $\mathrm{cm}$ & 5.75 & 5.5 \\
\hline Harmonic number & 5 & 3 \\
\hline X-ray wavelength, $\AA$ & 10 & 1 \\
\hline \multicolumn{3}{|l|}{ Coordinate slicing } \\
\hline Number of undulator periods & 100 & 100 \\
\hline$\sigma_{r}, \mu \mathrm{m}$ & 10.7 & 3.3 \\
\hline$\sigma_{r^{\prime}}, \mu \mathrm{rad}$ & 7.4 & 2.4 \\
\hline$\beta, \mathrm{m}$ & 2.5 & 2.5 \\
\hline$\sigma_{y}, \mu \mathrm{m}$ & 10 & 10 \\
\hline$\delta y\left(\sigma_{z}\right), \mathrm{mm}$ & 1.3 & 0.9 \\
\hline$U, \mathrm{MV}$ & 1.5 & 4.2 \\
\hline$A_{y}, \mathrm{~cm}$ & 2 & 1.2 \\
\hline \multicolumn{3}{|l|}{ Angular slicing } \\
\hline Number of periods & 30 & 100 \\
\hline$\sigma_{r}, \mu \mathrm{m}$ & 5.8 & 3.3 \\
\hline$\sigma_{r \prime}, \mu \mathrm{rad}$ & 13.6 & 2.4 \\
\hline$\beta, \mathrm{m}$ & 1 & 2.5 \\
\hline$\sigma_{y^{\prime}}, \mu \mathrm{rad}$ & 6.3 & 4 \\
\hline$\delta y^{\prime}\left(\sigma_{z}\right), \operatorname{mrad}$ & 1.4 & 0.4 \\
\hline$U, \mathrm{MV}$ & 2.5 & 5 \\
\hline$A_{y}, \mathrm{~cm}$ & 1.8 & 1.8 \\
\hline
\end{tabular}




$$
A_{y} \geq 20 \sigma_{y}+C O D+\delta y^{\prime}\left(7 \sigma_{z}\right) L_{u}
$$

where $A_{y}$ is the required vertical aperture for the A locations (first equation) and for the $\mathrm{B}$ locations (second equation), $C O D$ is the closed orbit distortion and $L_{u}$ is the length of the undulator. The first two terms in left parts Eqs.(7) represent the usual beam-stay-clear requirement, and the third term shows an extra aperture needed to accommodate the longitudinal-transverse coupling in the beam.

For an illustration of the above described technique we consider two examples of the coordinate slicing and two examples of the angular slicing presented in Table (I). These examples demonstrate what one can expect at synchrotron light sources similar to the Advanced Light Source and the Advanced Photon Source. The target for the optimization of the listed parameters was $350 \mathrm{fs}$ (FWHM) duration of the subpicosecond slice. We would like to emphasize that in both examples the requirement for the accelerating structure does not exceed what is achievable with the present technology (see for example [3]).

\section{X-ray optics and pulse compression}

So far two radiation sources one with the coordinate slicing and one with the angular slicing have been described. In this section we focus on the radiation source with angular slicing, assuming that the radiation source with the coordinate slicing can always be transformed by means of x-ray optics to a source with angular slicing.

In the following discussion it is assumed that the $x$-ray pulses are used as probes of a sample excited by a subpicosecond pump laser pulse. The response of the sample to the excitation can be measured in two ways. One can use the angle-time or coordinate-time correlation of the radiation for simultaneous observation of different time delays relative to the pump laser pulse. This can be done without time compression of the $\mathrm{x}$-ray pulse by detecting the individual time slices on a position sensitive detector after the sample (see Figure 2). For an $\mathrm{x}$-ray diffraction experiment the scattering may be performed in the horizontal plane to match the small horizontal divergence of the x-rays to the rocking curve of perfect crystals. With this approach, scanning the wavelength could be done by conventional means. Measurements 
could be performed either in a reflection or in a transmission geometry. In the reflection geometry the angle-time or coordinate-time correlation of the incident $x$-rays must be preserved after the sample.

Alternatively, one can compress the radiation of all beam slices into a single subpicosecond $\mathrm{x}$-ray pulse and use it to perform successive measurements at different time delays relative to the pump laser pulse. Asymmetrically-cut crystals may be used as optical elements for x-ray pulse compression [4]. Because of the different incident and diffractive angles, they produce a variable path length across the x-ray beam. After a sagittal collimating mirror, two asymmetrically-cut crystals can act as monochromator and pulse compressor for $x$-rays with angular slicing (see Figure 3). In this case the optical path length difference $\delta \ell$ in the vertical scattering plane is given by,

$$
\delta \ell \simeq 2 \frac{r \delta y^{\prime}}{\sin (\theta-\alpha)} \sin \theta \sin \alpha\left(1+\frac{\sin (\theta+\alpha)}{\sin (\theta-\alpha)}\right),
$$

where $r$ is the source distance to the collimating mirror, $\theta$ is the Bragg angle and $\alpha$ is the asymmetry angle between the Bragg planes and the crystal surface. The effect of the finite vertical source size gives less than $10 \mu \mathrm{m}$ pulse lengthening for a typical set of parameters. As seen in Eq.(8), the magnitude of the x-ray pulse compression depends on the Bragg angle $\theta$. Consequently, each $x$-ray wavelength would require a different asymmetrically-cut crystal. Scanning a small range of wavelength $(\sim 10 \%)$ would be possible by compensating the variation of path length with a small change in the RF voltage $U$. A practical example of $\mathrm{x}$-ray pulse compression for $1 \AA$ wavelength is given in Table II.

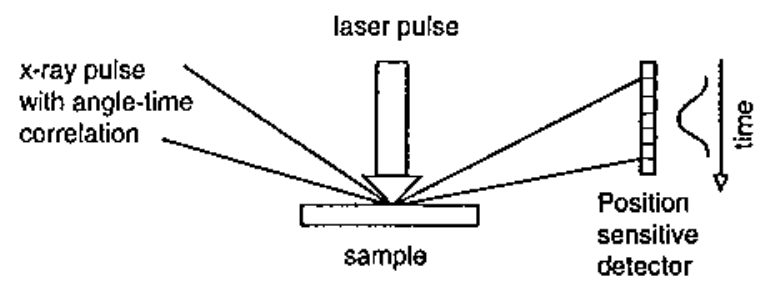

Figure 2: Schematic diagram of an angle-time correlated $x$-ray pulse incident on a sample. A position sensitive detector is used to obtain a set of laser pump / $\mathrm{x}$-ray probe delay times. 


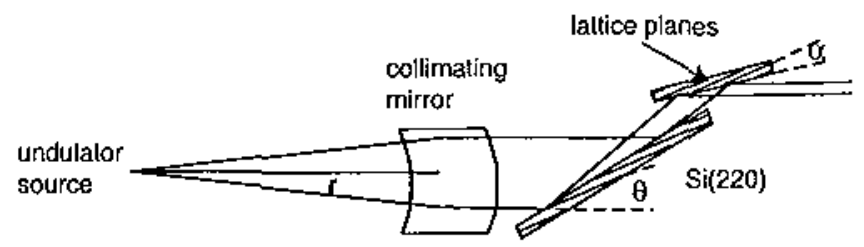

Figure 3: Optical scheme for pulse compression with a collimating mirror and a double asymmetrically-cut crystal monochromator.

Conducting pump and probe experiments on a subpicosecond time scale requires an adequate synchronization of the laser pump pulse to the electron bunch circulated in the storage ring. At present, the synchronization of a 10fs-Ti:Sapphire laser to an electron beam with a jitter of 0.9 ps (FWHM) has been demonstrated [5]. The requirement for jitter may be relaxed with the technique using the position sensitive detector. With the detector producing a new data array for each laser flash, time jitter can possibly be taken into account in the off-line analysis.

Table II: Parameters for $\mathrm{x}$-ray pulse compressor using asymmetrically-cut crystals.

\begin{tabular}{|l|c|}
\hline Crystals & $\mathrm{Si}(220)$ \\
X-ray wavelength, $\stackrel{\AA}{ }$ & 1 \\
Source distance, r, m & 40 \\
$\theta$, degree & 15 \\
$\alpha$, degree & -10 \\
$\delta y \prime$, mrad & 0.4 \\
$\delta \ell, \mathrm{cm}$ & 0.4 \\
\hline
\end{tabular}

\section{Flux and brightness}

The longitudinal-transverse coupling induced in the electron bunches by the $R F$ cavities does not affect how the electrons radiate in the undulators. They emit the same number of $x$-ray photons as they would emit without coupling. However, because the radiation of all subpicosecond slices of the electron 
bunch is spatially separated, the brightness of the radiation is reduced:

$$
\mathcal{B}=\mathcal{B}_{0} / M,
$$

where $\mathcal{B}$ and $\mathcal{B}_{0}$ is the source brightness with and without spatial separation of the subpicosecond slices.

Continuing the examples from Section 2, we present in Table III the number of $\mathrm{x}$-ray photons per pulse produced in a given undulator harmonic. Then, we calculate the average flux of the $\mathrm{x}$-rays and the brightness.

Table III: Flux and brightness

\begin{tabular}{|l|c|c|}
\hline Time resolution (FWHM), fs & 350 & 350 \\
Beam energy, GeV & 1.5 & 6.0 \\
Number of electrons/bunch & $1 \times 10^{10}$ & $1 \times 10^{10}$ \\
Bunch frequency, MHz & 250 & 125 \\
X-ray wavelength, $\AA$ & 10 & 1 \\
Harmonic number & 5 & 3 \\
\hline Coordinate slicing & & \\
\hline Photons/pulse (0.1\%bw) & $4 \times 10^{5}$ & $2 \times 10^{6}$ \\
Flux, photons $/ \mathrm{sec}^{-1}(0.1 \% \mathrm{bw})^{-1}$ & $1 \times 10^{14}$ & $2.5 \times 10^{14}$ \\
$\mathcal{B}, \mathrm{s}^{-1} \mathrm{~mm}^{-2} \mathrm{mrad}^{-2}(0.1 \% \mathrm{bw})^{-1}$ & $7 \times 10^{18}$ & $1.7 \times 10^{19}$ \\
\hline Angular slicing & & \\
\hline Photons/pulse $(0.1 \% \mathrm{bw})^{-1}$ & $1 \times 10^{5}$ & $2 \times 10^{6}$ \\
${\text { Flux, photons } / \mathrm{sec}^{6}(0.1 \% \mathrm{bw})^{-1}}$ & $3 \times 10^{13}$ & $2.5 \times 10^{14}$ \\
$\mathcal{B}, \mathrm{s}^{-1} \mathrm{~mm}^{-2} \mathrm{mrad}^{-2}(0.1 \% \mathrm{bw})^{-1}$ & $2 \times 10^{18}$ & $1.7 \times 10^{19}$ \\
\hline
\end{tabular}

X-ray pulses generated in the storage ring can be utilized in pump and probe measurements with lasers. The average laser power has a certain limit, and for a given pulse energy this limit defines the maximum repetition rate of the laser pulses (much less than the bunch repetition rate as shown below).

For illustrative purposes, consider an absorption experiment at either 10 $\AA$ or $1 \AA$ wavelength. It may be assumed that the sample is illuminated with a laser fluence of $\sim 50 \mathrm{~mJ} / \mathrm{cm}^{2}$. The radiation source may be imaged to the sample with 1:1 magnification. This gives an x-ray spot size of approximately $2 \times 10^{-5} \mathrm{~cm}^{2}$ (FWHM) in both cases. If the laser beam is focused to twice as large an area in order to provide more uniformity of the laser fluence, a laser 
pulse of approximately $2 \mu \mathrm{J}$ is required. For a $1 \mathrm{~W}$ laser this corresponds to a repetition rate of $500 \mathrm{kHz}$.

Thus, in the examples shown in Table III, the flux of $x$-ray photons that can be utilized in pump and probe experiments is $2 \times 10^{11}$ photons/sec $(0.1$ $\% \mathrm{bw})^{-1}$ for $10 \AA$ wavelength and $1 \times 10^{12} \mathrm{x}$-ray $/ \mathrm{sec}(0.1 \% \mathrm{bw})^{-1}$ for $1 \AA$ wavelength.

\section{Acknowledgments}

We are grateful to H. Padmore and B. Kincaid for useful discussions and to $\mathrm{S}$. Chattopadhyay for his continuous encouragement.

\section{References}

[1] O.A. Nezhevenko, A.A. Zholents, Proc. of the XIII-th Inter. Conf. on High Ener. Acc., v.1, p.70 (1986).

[2] R. Palmer, SLAC-PUB-4107 (1988).

[3] K. Akai, et al., Proc. of the 1993 Part. Acc. Conf. IEEE, v.2, p.769 (1993).

[4] T. Matsushita and H. Hashizume, Handbook of Synchrotron Radiation v.1, ed. E.E. Koch, p.261, North-Holland, Amsterdam (1993).

[5] G.M.H.Knippels, et al., abstract to 20th Int. FEL98 Conf., Aug 16-21, 1998. 